\title{
Hydrogen Technologies
}

\section{Photobiological Production of Hydrogen}

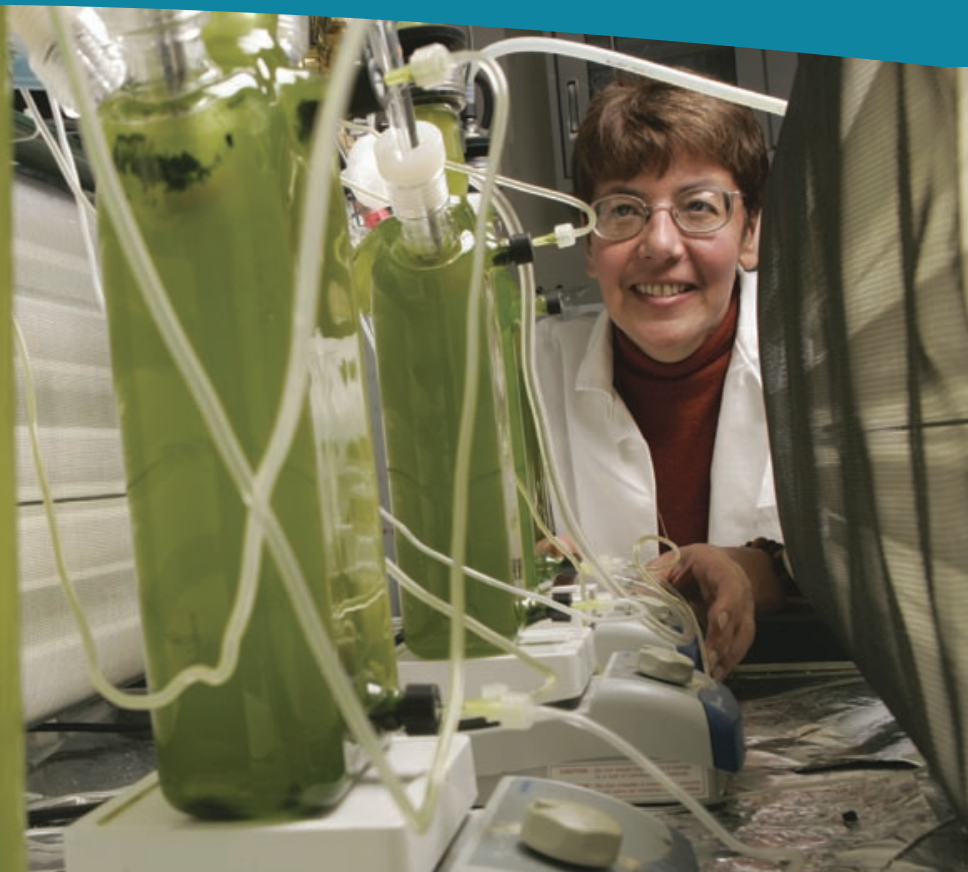

A researcher works on a novel way to use green algae to produce hydrogen directly from water and sunlight. PIX14581

\section{Highlights}

Certain algae and cyanobacteria photoproduce hydrogen for short times as a way to get rid of excess energy before starting up the photosynthetic carbon fixation process.

NREL researchers have successfully developed a bacterial system for synthesis of a key enzymehydrogenase - that is responsible for photosynthetic hydrogen evolution in green algae.

They are now focusing on enzyme engineering to block the access of oxygen, which can stop hydrogen production, to the catalytic site of the hydrogenase.

Photobiological production of hydrogen has potential to be one of the most cost effective ways to produce hydrogen from renewable energy.

\section{What do these things have in common: a few minutes' lag before algae can} start making sugar from photosynthetic energy; narrowing passageways within an enzyme; and a Mexican restaurant in Denver?

The answer is that they all are related in some way to efforts by National Renewable Energy Laboratory (NREL) scientists to develop technology for photobiological production of hydrogen - allowing the direct capture of renewable energy as an ideal, nonpolluting, non-greenhouse-gas-producing transportation fuel. In one of nature's quirks, certain algae and cyanobacteria photoproduce hydrogen for short times as a way to get rid of excess energy. NREL researchers are seeking to turn that quirk into technology for directly converting solar energy to transportation fuel-by overcoming the oxygensensitivity trigger that shuts off the hydrogen production process in green algae, by doing the same for cyanobacteria, and by directing green algae toward the process by depriving them of the nutrition needed for normal photosynthesis.

Hydrogen use in fuel cells produces only water vapor and electricity at the point of use. Also, hydrogen can be stored to match energy production to energy demand. These make the use of hydrogen highly attractive as an energy carrier in addition to, or instead of, electricity. As an energy carrier rather than a basic energy source, however, hydrogen is only as "good" as the energy used to make it. Nearly all current U.S. and most of the world's hydrogen production involves steam reformation of natural gas. This, of course, uses an increasingly scarce fossil fuel that can be used directly for electrical production to meet peak demand and also has home heating as a high-priority use. Hydrogen could also be made by reforming gasified biomass or using renewably generated energy to power water electrolysis. But in the long run, the highest efficiencies should be achieved with technologies such as photoelectrochemistry or photobiochemistry, which can produce hydrogen directly from solar energy. 


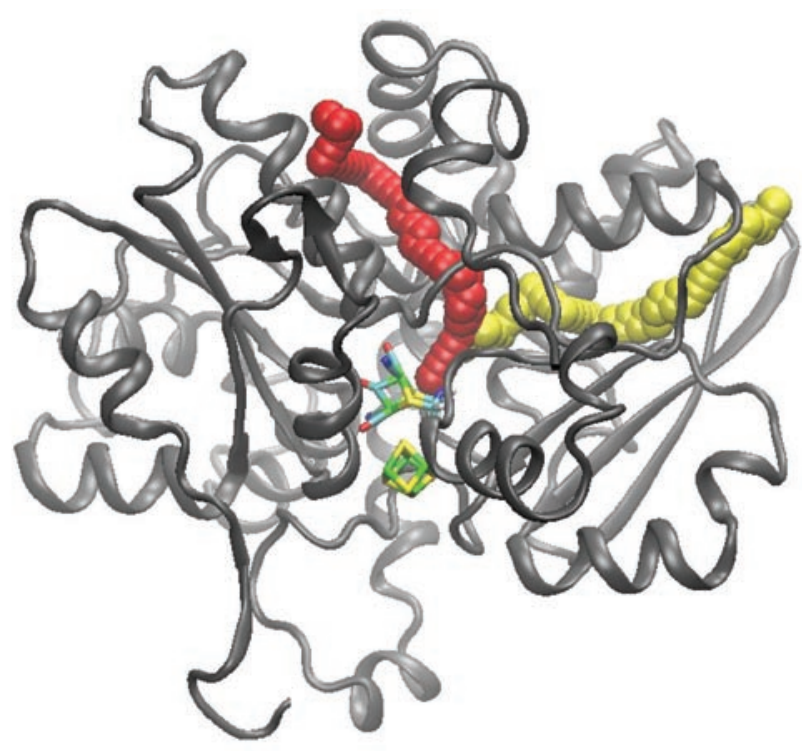

Visualization of the oxygen-diffusion channels (red and yellow ribbons) in the hydrogenase enzyme structure. The oxygen-sensitive FeS catalytic centers are indicated in green, yellow and red, and the backbone of the protein is shown in gray.
Hydrogen is essentially an energy carrier rather than a primary fuel. Although the most abundant element, it cannot be mined. It rarely exists in elemental form, instead being bound up in water or other compounds. It does, however, sometimes exist in nature, most notably within certain algae and photosynthetic bacteria that produce it as a way to dissipate excess energy. If we can effectively manipulate those microorganism processes, we can capture solar energy directly in a form that is an ideal transportation fuel. NREL scientists are researching these hydrogen production processes to find ways to keep them turned on instead of being only temporary, as they are naturally. Two main current research paths involve taking advantage of the hydrogen-production process in green algae by either overcoming oxygen inhibition of the process or by using nutrient deprivation to manipulate the algae into using the process. A third research avenue seeks to overcome oxygen inhibition in a cyanobacterium.

\section{Overcoming Oxygen Inhibition in Green Algae}

Algal photosynthesis and hydrogen production are sister processes. Both start with the same solar-energy-activated splitting of water to oxygen, electrons, and protons; protons and electrons then go to a second enzymatic reaction. In one case, the "normal" second reaction fixes carbon dioxide to produce sugar, and in the other, an alternative reaction produces hydrogen molecules. Picture a population of algae at the bottom of a pond at night. The organisms have been respiring and not photosynthesizing, so conditions have become largely anaerobic. When light first hits, the water-splitting reaction starts up right away. But there is a lag time of a few minutes in the carbon-fixing reaction before light activates the enzymes for carbon fixation. Electrons produced by the water splitting would harm or destroy the organism if the excess energy were not somehow dissipated. The algae evolved an alternative second reaction that combines the protons and electrons to form hydrogen molecules, thus getting rid of the excess energy. It is this temporary alternative process-which normally only lasts for a few minutes-that we would like to tap. Once enough oxygen builds up from the water-splitting reaction, the algae are forced to shut off hydrogen production and go to carbon fixation to the starch that provides their food source.

Thus, algal hydrogen production is naturally inhibited by the presence of oxygen. Overcoming that inhibition is a major focus of photobiological hydrogen production research. The algal hydrogen-production process is driven by enzymes known as [Fe-Fe] hydrogenase, because of the presence of a unique $2 \mathrm{Fe} 2 \mathrm{~S}$ (iron and sulfur) metallo-cluster in the catalytic center of the core of the proteins.

Researchers have found that the shut-off of hydrogen production is due to inhibition of the enzyme by oxygen. The inhibition depends on oxygen physically diffusing into the enzyme's catalytic center and irreversibly binding to it, thus halting further catalytic activity. NREL scientists and research partners have built computer models of the [Fe-Fe]-hydrogenase from the bacterium Clostridium pasteurianum (not photosynthetic, but with a structure very similar to that of the hydrogenase of the green alga Chlamydomonas reinhardtii upon which NREL research centers: Clostridium's crystal structure is known; Chlamydomonas' structure has not yet been solved). This model allowed them to conduct highly sophisticated simulations of gas movement in and out of the enzyme. They found that, although hydrogen may move out of the enzyme by additional pathways, there are just two main ones by which oxygen molecules could make their way into the enzyme. 
With this knowledge, NREL researchers were able to model amino-acid substitutions and other potential mutations and combinations of mutations along the pathways to identify promising ones for preventing oxygen from reaching the enzyme center. They then proceeded to actually enzyme engineer some of those mutations, express them in the industrial bacterium E. coli (again, not itself photosynthetic) and then test the oxygen sensitivity and hydrogen productivity of the recombinant enzymes. Thus far, although one of many attempted mutations along one enzyme pathway yielded modest improvement, others did not, proving detrimental for both oxygen sensitivity and hydrogen productivity. Hydrogenase engineering efforts for the enzyme continue, as this holds great promise as the key to overcoming oxygen inhibition. An analysis projects that this could lead to hydrogen production with as high as 10\% efficiency in the conversion of solar energy.

\section{Inactivating Algal Photosynthesis by Nutrient Deprivation}

An alternative approach to sustaining algal hydrogen production artificially is to partially inactivate the normal photosynthetic process. NREL scientists have been able to do this by depriving an algal culture of sulfate, which is necessary for protein synthesis and particularly for the production of a key enzyme for photosynthesis that has an especially fast turnover time. As the sulfate is used up, photosynthesis slows. After about a day, photosynthesis produces less oxygen than respiration consumes, and the culture becomes anaerobic and switches from carbon fixation to a combination of hydrogen-production and starch degradation. Starch degradation supports the consumption of the low amount of oxygen that is still derived from residual photosynthesis and contributes reductants to hydrogen production. So, if the culture is present in a sealed reactor, it starts accumulating hydrogen gas.
NREL's development of this technique has already progressed through several advances. Initial operation with the algae in simple suspension could be maintained for 3-4 days. The cycle was repeated at least three times by alternating with periods of providing sulfate for normal photosynthesis to allow the algae to provide energy for themselves and avoid other problems from sulfur deprivation.

The next level of development used a chemostat-based system that cultivated the algae with adequate nutrients in one reactor. The algae were then transferred to a second, sulfate-deprived reactor in which they produced hydrogen. Finally, in the continuous flow system, the algae are used as feedstock for fermentative processes or gasified. This system cut costs to one-third as much as simple batch processing and was run continuously for 6 months.

The next improvement came with immobilization of the algal cultures on either glass fibers or alginate films. Because the immobilized algae were no longer swimming around or performing other functions, they needed less energy (algae live partially off stored starch while in the hydrogen-producing state) and the culture could be maintained for 25 days in batch operation. An alternate version of the immobilization technique that provides enough nutrients for minimal starch production, but not enough to shut off the hydrogen production, was operated continuously for 3 months.

Because nutrient deprivation inherently limits the productivity of the algae, this technology is likely to achieve 1\%-2\%

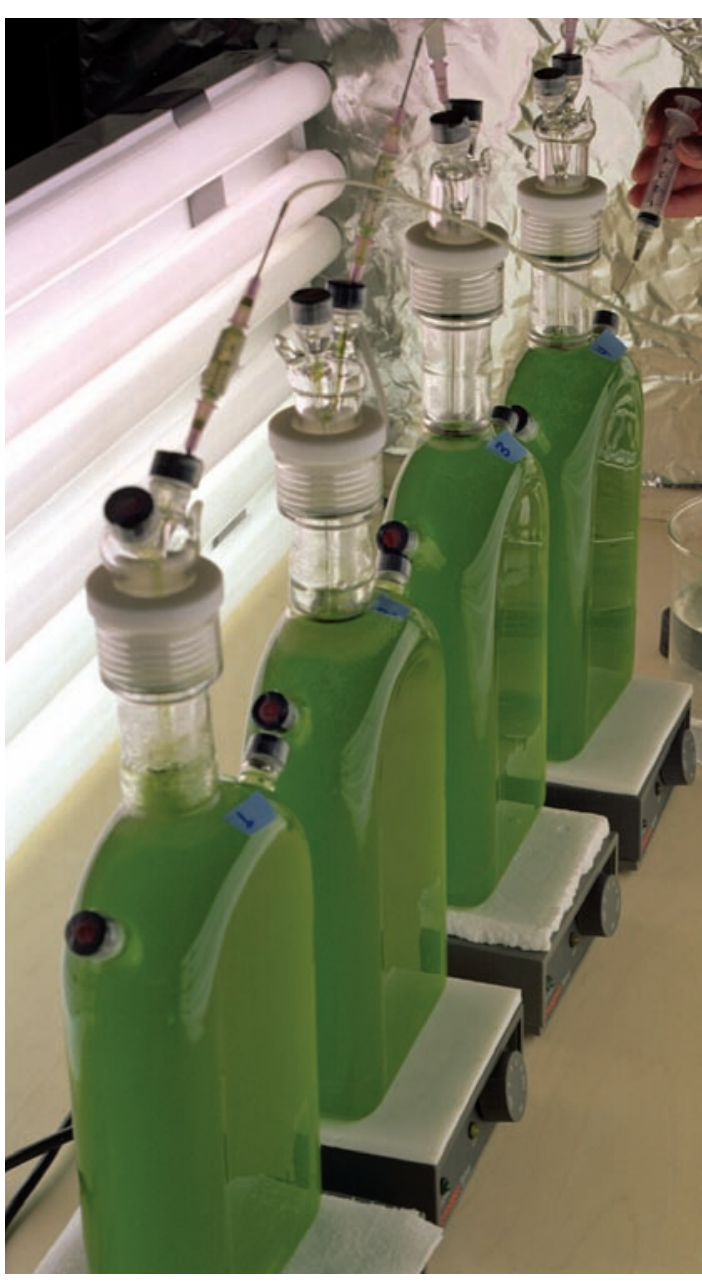

A set of bioreactors used for photobiological hydrogen production by green algae. PIX 08740 solar energy capture at best, hence our recent emphasis on engineering the hydrogenase enzyme. Nevertheless nutrient deprivation has proved highly valuable to NREL researchers in developing an understanding of algal hydrogen production metabolism and potential methods for cultivation. 


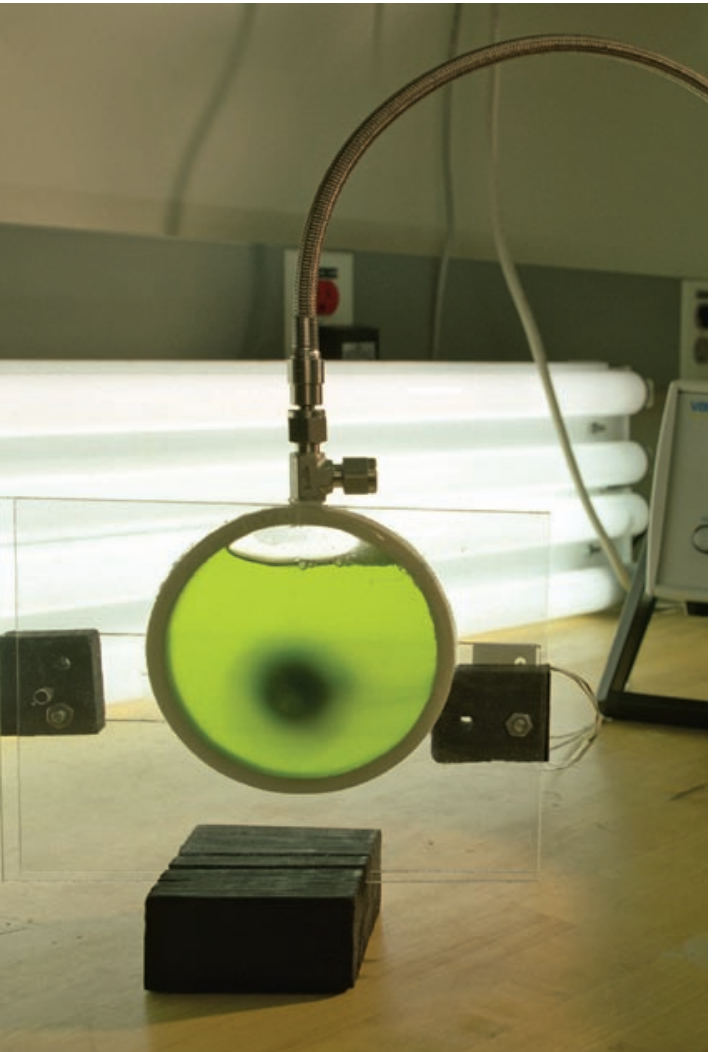

Algal hydrogen production. PIX 09839

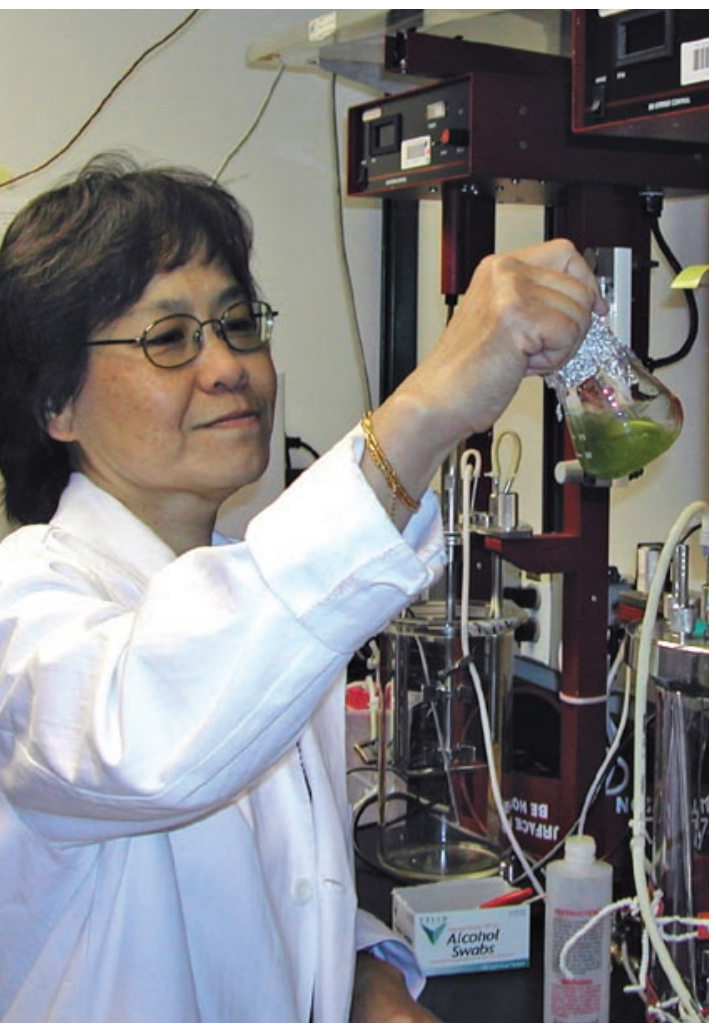

A researcher examines a natural cyanobacterial culture for hydrogen production using sunlight and water in a bench top bioreactor. PIX 15277

\section{Overcoming Oxygen Inhibition in Cyanobacteria}

Cyanobacteria, also known as bluegreen algae, have characteristics of both algae and bacteria. The algal characteristics include being photosynthetic and producing hydrogen for a short time when starting photosynthesis, much as green algae do. The cyanobacteria have a [Ni-Fe] hydrogenase rather than a $[\mathrm{Fe}-\mathrm{Fe}]$ hydrogenase, however. The nickel-iron enzyme is somewhat less sensitive to oxygen and its inhibition is reversible-the green algae have to synthesize additional enzyme every time their [FeFe] hydrogenase is irreversibly inhibited by oxygen-suggesting the cyanobacteria might have advantages for industrial hydrogen production. It was discovered only recently that cyanobacteria could be made to produce hydrogen from photosynthetic water oxidation.

Researchers outside NREL have successfully demonstrated hydrogen production with a strain of Synechosystis that they engineered to be deficient in a protein needed for a competing reaction.

NREL researchers, meanwhile, identified an oxygen-tolerant hydrogenase in a nonoxygenic photosynthetic bacterium, Rubrivivax gelatinosus CBS. The "CBS" appellation coming from the name of a Denver restaurant in whose parking lot researchers found the bacterium while looking for organisms that produce hydrogen through the oxidation of carbon monoxide. They have now successfully cloned and sequenced the CBS hydrogenase genes and are working to transfer them to the mutant Synechocystis. As with green algae, successfully overcoming oxygen inhibition of hydrogen production by cyanobacteria could lead to industrial processes yielding as much as $10 \%$ solar energy capture, so this is a very exciting research avenue.

All these efforts to overcome the natural short-term nature of algal hydrogen production are still in early research stages. Many challenges face any industrial production process, but these have promise for producing an ideal transportation fuel in a renewable and nonpolluting way.

\section{Contacts}

NREL Public Relations

$303-275-4050$

NREL Technology Transfer

Tom Williams, 303-275-4485

Hydrogen Technologies and Systems Center Robert Remick, 303-275-3830

Photobiological Hydrogen Research

Maria Ghirardi, 303-384-6312
Hydrogen Technologies

National Renewable Energy Laboratory

1617 Cole Boulevard, Golden, Colorado 80401-3393

303-275-3000 • www.nrel.gov

Operated for the U.S. Department of Energy

Office of Energy Efficiency and Renewable Energy

by Midwest Research Institute - Battelle

NREL/FS-560-42285 • Revised November 2007

Printed with a renewable-source ink on paper containing at least

$50 \%$ wastepaper, including $10 \%$ post consumer waste. 